\title{
Pharmacogenomics Study for Raloxifene in Postmenopausal Female with Osteoporosis
}

\author{
Hsing-Fang Lu, ${ }^{1}$ Po-Hsin Chou, ${ }^{2,3}$ Gan-Hong Lin, ${ }^{4}$ Wan-Hsuan Chou, ${ }^{1}$ Shih-Tien Wang, ${ }^{2,3}$ \\ Wirawan Adikusuma $\mathbb{D}^{1,5}$ Eko Mugiyanto, ${ }^{6}$ Kuo-Sheng Hung $\mathbb{D}^{4,7,8}$ \\ and Wei-Chiao Chang ${ }^{1,4,9,10}$ \\ ${ }^{1}$ Department of Clinical Pharmacy, School of Pharmacy, Taipei Medical University, Taipei, Taiwan \\ ${ }^{2}$ School of Medicine, National Yang-Ming University, Taipei, Taiwan \\ ${ }^{3}$ Department of Orthopedics and Traumatology, Taipei Veterans General Hospital, Taipei, Taiwan \\ ${ }^{4}$ Clinical Pharmacogenomics and Pharmacoproteomics, School of Pharmacy, Taipei Medical University, Taipei, Taiwan \\ ${ }^{5}$ Department of Pharmacy, University of Muhammadiyah Mataram, Mataram, Indonesia \\ ${ }^{6}$ Clinical Drug Development of Chinese Herbal Medicine, School of Pharmacy, Taipei Medical University, Taipei, Taiwan \\ ${ }^{7}$ Graduate Institute of Injury Prevention and Control, College of Public Health, Taipei Medical University, Taipei 11042, Taiwan \\ ${ }^{8}$ Department of Neurosurgery, Taipei Medical University-Wan Fang Hospital, Taipei, Taiwan \\ ${ }^{9}$ Pain Research Center, Wan Fang Hospital, Taipei Medical University, Taipei, Taiwan \\ ${ }^{10}$ Biotechnology Research and Development, College of Pharmacy, Taipei Medical University, Taipei, Taiwan
}

Correspondence should be addressed to Kuo-Sheng Hung; kshung25@gmail.com and Wei-Chiao Chang; wcc@tmu.edu.tw

Received 19 May 2020; Revised 25 June 2020; Accepted 7 August 2020; Published 31 August 2020

Academic Editor: Wen-Jun Tu

Copyright ( $\odot 2020$ Hsing-Fang Lu et al. This is an open access article distributed under the Creative Commons Attribution License, which permits unrestricted use, distribution, and reproduction in any medium, provided the original work is properly cited.

Osteoporosis is characterized by decreased bone mineral density and increased risk of fracture. Raloxifene is one of the treatments of osteoporosis. However, the responses were variable among patients. Previous studies revealed that the genetic variants are involved in the regulation of treatment outcomes. To date, studies that evaluate the influence of genes across all genome on the raloxifene treatment response are still limited. In this study, a total of 41 postmenopausal osteoporosis patients under regular raloxifene treatment were included. Gene-based analysis using MAGMA was applied to investigate the genetic association with the bone mineral density response to raloxifene at the lumbar spine or femoral neck site. Results from gene-based analysis indicated several genes (GHRHR, ABHD8, and TMPRSS6) related to the responses of raloxifene. Besides, the pathways of iron ion homeostasis, osteoblast differentiation, and platelet morphogenesis were enriched which implies that these pathways might be relatively susceptible to raloxifene treatment outcome. Our study provided a novel insight into the response to raloxifene.

\section{Introduction}

Osteoporosis is defined as the weakened architecture of the bone and elevated risk of fracture [1]. In Taiwan, the prevalence of osteoporosis is about $6.9 \%$ in males over the age of 65 and $21.2 \%$ in postmenopausal females [2]. Several risk factors of osteoporosis have been identified, including menopause, dairy intake, life style, and genetic factors [3]. Osteoporosis is a polygenic disease that is determined by multiple genetic effects from several genes. Variants near
WNT16 and FONG have been reported to be associate with bone mineral density (BMD) [4-7]. The risk of osteoporosis increases dramatically in the elderly population, that bone fractures are known to increase mortality and lead to an enormous healthcare burden on society $[8,9]$. Since the aged population is growing rapidly, osteoporosis has become a crucial clinical issue.

Nonsteroidal selective estrogen-receptor modulators (SERMs) can inhibit bone resorption. SERMs have been widely used in the prevention and treatment of postmenopausal 
osteoporosis [10]. Raloxifene is a SERM agent used in the clinic for postmenopausal osteoporosis [11]. The drug is a partial agonist for the estrogen receptor and is known to inhibit proliferation of breast epithelium, while preserving bone mineral density (BMD). This drug increases BMD and reduces the occurrences of new vertebral fractures [12]. However, the therapeutic response of raloxifene varies widely among patients. Previous studies revealed that two polymorphisms of ESR1 (estrogen receptor 1) (rs9340799 and rs2234693) are associated with the BMD response to raloxifene in osteoporosis patients $[13,14]$. UGT1A8 (UDP glucuronosyltransferase family 1 member A8) encodes an enzyme involved in extrahepatic glucuronidation of raloxifene [15], and UGT1A $8 * 2$ was found to be associated with more raloxifene glucuronide metabolite formation in human jejunum tissue [16]. Labad et al. also identified a missense variant of UGT1A8, rs1042597, that associated with the treatment responses to raloxifene in postmenopausal schizophrenic women [17].

Previous studies focused on the association between therapeutic responses and variants of a single gene. This type of study design called a candidate gene approach, which is a hypothesis-driven method that tests a small number of variants [18]. Limited genetic variants are unable to discover novel mechanisms. On the other hand, genome-wide association study (GWAS) is a hypothesis-free or hypothesisgenerating method that provides opportunities to identify novel insights into the phenotype. In addition, GWAS is able to explore new genetic factors of complex human traits [19]. Since the mechanism of how genes influence response of raloxifene remains unclear, the aim of the current study is using gene-based analysis to identify the main network correlated with the responses of raloxifene.

\section{Materials and Methods}

2.1. Sample Collection. We collected samples from the neurosurgery outpatient clinic in Wang-Fang Hospital, Taipei, Taiwan. Our inclusion criteria were as follows: (1) 60 to 85-yearold postmenopausal female, (2) $T$-score $\leqq-2.5$, and (3) under regular raloxifene treatment $(60 \mathrm{mg} /$ day). Patients with continuous steroid use (of over 6 months) or longterm inflammatory disease were excluded. In total, 41 osteoporosis patients were recruited. Bone mineral density (BMD) was measured by dual-energy radiograph absorptiometry (Lunar Prodigy, version 9.1, GE Healthcare, Madison, WI, USA) with standard protocols at the lumbar spine (LS; L2-4 or L1-4) and femoral neck (FN). Age, gender, body mass index (BMI), medication profile, and follow-up duration of all individual were collected. We also collected the BMD profiles after patients receiving treatment for 1-2 years. The study was approved by the Joint Institutional Review Board of Taipei Medical University. All subjects provided written informed consent.

2.2. DNA Extraction and Genotyping Array. Peripheral whole blood was collected from the patients. Gentra Puregene Blood Kit (Qiagen, Valencia, CA, USA) was used to extract the genomic DNA. The extraction process was according to the user manual of the product. The quality of DNA was confirmed by $1 \%$ agarose gel electrophoresis. Sample with no obvious degradation can be submitted to further analysis. Affymetrix Axiom TWB SNP Array (Thermo Fisher Scientific, USA) was used to perform GWAS. The process was done by the National Center for Genome Medicine, Taiwan (NCGM).

2.3. Quality Control. PLINK [20] was applied to conduct the quality control of genotype data. We excluded SNPs with low genotyping call rate $(<0.95)$, minor allele frequency less than 0.05 , and $P$ value of Hardy-Weinberg equilibrium less than $1 \times 10^{-6}$. Individuals with a sample call rate less than 0.95 were excluded. The imputation task was done by Michigan Imputation Server (Minimac3). Data from the Haplotype Reference Consortium (HRC) was used as reference panels for imputation in order to increase statistical power. After imputation, only variants which $\mathrm{MAF} \geqq 0.05$ and $R^{2} \geqq 0.3$ were included in the association analysis. Principle components (PCs) were generated by PLINK with samples from HapMap3. A linear regression model was used for statistically testing the associations between SNPs and phenotypes by PLINK2. We defined the percentage change of bone mineral density from baseline by the following equation:

$$
x=\frac{2^{\text {nd }} B M D-1^{\text {st }} B M D}{1^{\text {st }} B M D} .
$$

We further normalized phenotype before association analysis. We included age, BMI, follow-up months, and PC1 to PC10 as covariates in the analysis model. The Manhattan plot and qq plot were visualized by the $\mathrm{R}$ package "qqman," and the suggestive significant threshold was $1 \times$ $10^{-5}$ as the default setting of this package. The regional plots were generated by LocusZoom [21].

2.4. Variant Annotation. We annotated the rs number of candidate variates by ANNOVAR [22] with avsnp150 database. The annotation of nearby genes was according to the NCBI RefSeq hg19 reference panel. In order to compare our results with previous studies, the data of GWAS catalog v1.0.2 (20190322) was downloaded from GWAS catalog website.

2.5. Gene-Based Analysis and Gene-Set Analysis. Since our sample size was very limited, the effects of individual markers may be too weak to detect. Therefore, we applied MAGMA to conduct gene-based analysis to increase the power [23]. This method can analyze multiple genetic markers together to determine their joint effect. Input SNPs were mapped to 18306 protein coding genes; therefore, the Bonferroni significant threshold for gene analysis was using $P<2.73 \times 10^{-6}$. To understand the biological enriched pathways related with the raloxifene treatment response, we used gene-set analysis by MAGMA. The curated gene sets and GO terms were obtained from MsigDB v6.1. Curated gene sets were collected from 9 data resources including KEGG, Reactome, BioCarta, and biomedical literatures. Variates located within transcription start region and stop region of the gene region will be 


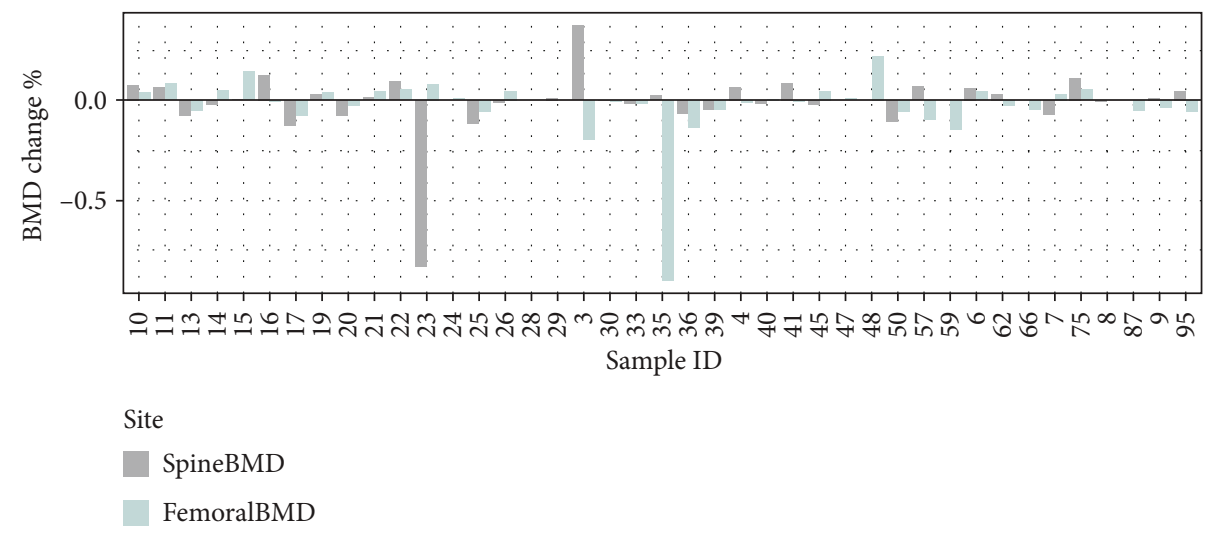

FIgURE 1: The distribution of the BMD change $\%$ at the lumbar spine and femoral neck site.

mapped to that gene. We used 1000 genomes phase 3 as reference genome.

\section{Results}

3.1. Baseline Characteristics. The distribution of the BMD change percent is shown in Figure 1. After treatment, the $\mathrm{BMD}$ response to raloxifene was diverse at both lumbar spine and femoral neck sites in patients. In total, 41 patients were assessed for femoral neck BMD (S Table 1), and the average percentage of BMD change was $-2.5 \pm 15.8 \%$. The mean follow-up time was $35.61 \pm 23.54$ months among all subjects. The mean age of subjects was $72.4 \pm 7.0$ years, and the average BMI was $23.96 \pm 3.49 \mathrm{~kg} / \mathrm{m}^{2}$. Among the 41 patients assessed for FN BMD, 34 were also assessed for lumbar spine BMD. The average age and BMI of this subset were similar to the complete group.

3.2. Association Analysis. We conducted a genome-wide association analysis to reveal the risk loci associated with raloxifene treatment response. A principal component analysis showed that there was no population stratification (S Figure 1). Neither site revealed genome-wide significant association signal of BMD change (S Figures 2, 3). However, there were 112 variants with suggestive significance for LS percent BMD change, while 13 variants showed significance for FN percentage $\mathrm{BMD}$ change. The most significant variant in the LS site was $\operatorname{rs} 7768089\left(P=2.29 \times 10^{-6}\right)$, which was an intronic SNP of FUT9 located on 6q16.1. Meanwhile, rs34311394 at $12 \mathrm{q} 21.2$ was the most significant $\operatorname{SNP}(P=$ $4.44 \times 10^{-6}$ ) for the FN site. This SNP is located in the intergenic region between CSRP2 and E2R7. There was no overlap variants among suggestively significant SNPs between the two sites.

3.3. Candidate Genes Cause Decreased BMD in Mutant Mice. Next, a gene-based analysis was conducted by MAGMA. However, none of the genes reached the threshold of statistical significance (Figure 2). The top ten significant genes at the LS and FN sites are shown in Tables 1 and 2. To understand the potential biological functions of these genes, we queried the phenotypes of mutant mice in the International Mouse Phenotype Consortium (IMPC) database.
Among the queried genes, GHRHR, ABHD8, and TMPRSS6 showed decreased BMD in a homozygous mutant mouse in both sexes.

3.4. Enrichment of Osteoblast Differentiation Pathway. We further applied gene-set analysis to reveal the significant enriched gene sets. After FDR adjustment, although none of the pathway achieved the statistical significance (Tables 3 and 4$)$, iron homeostasis for the LS site $\left(P=2 \times 10^{-4}\right)$ was the most significant gene set. Regarding the femoral neck site, the most significant gene set was platelet morphogenesis $\left(P=1 \times 10^{-4}\right)$. In addition, another gene sets of "osteoblast differentiation by phenylamil up" showed nominal significance for the femoral neck site as well $\left(P=4 \times 10^{-4}\right)$.

3.5. Comparison with Previous Candidate Variants. Since some variants have been reported to be associated with response to raloxifene, we next focused two SNPs within ESR1 (rs9340799 and rs2234693) and one SNP in UGT1A8 (rs1042597). As shown in Supplementary Table 2, none of the SNPs demonstrated nominal significance $(P>0.05)$ at the LS or FN site. We further extracted larger regions around these two genes $( \pm 500 \mathrm{Mb})$ of two phenotypes and generated regional plots. However, no variant within these regions reached suggestive significance (S Figure 4-7).

\section{Discussion}

This study used two skeletal positions to assess the relationships between BMD response to raloxifene and gene network. Although statistical power was limited sample size, several candidates including GHRHR, ABHD8, and TMPRSS6 still addressed significance after gene-based analysis. These three genes were also associated with decreased BMD in mutant mouse models. From gene-set analysis, we found that iron homeostasis and osteoblast differentiation gene sets were enriched. Previous studies indicated that iron overload affects the bone phenotype and increases risk of bone fracture. Iron overload is an important factor that is involved in the activation of osteoclast, the differentiation process, and the reduction of osteoblast formation $[24,25]$. Besides, in patients with thalassemia major, which is often characterized by low BMD values and iron overload, the circulating IGF-1 levels 


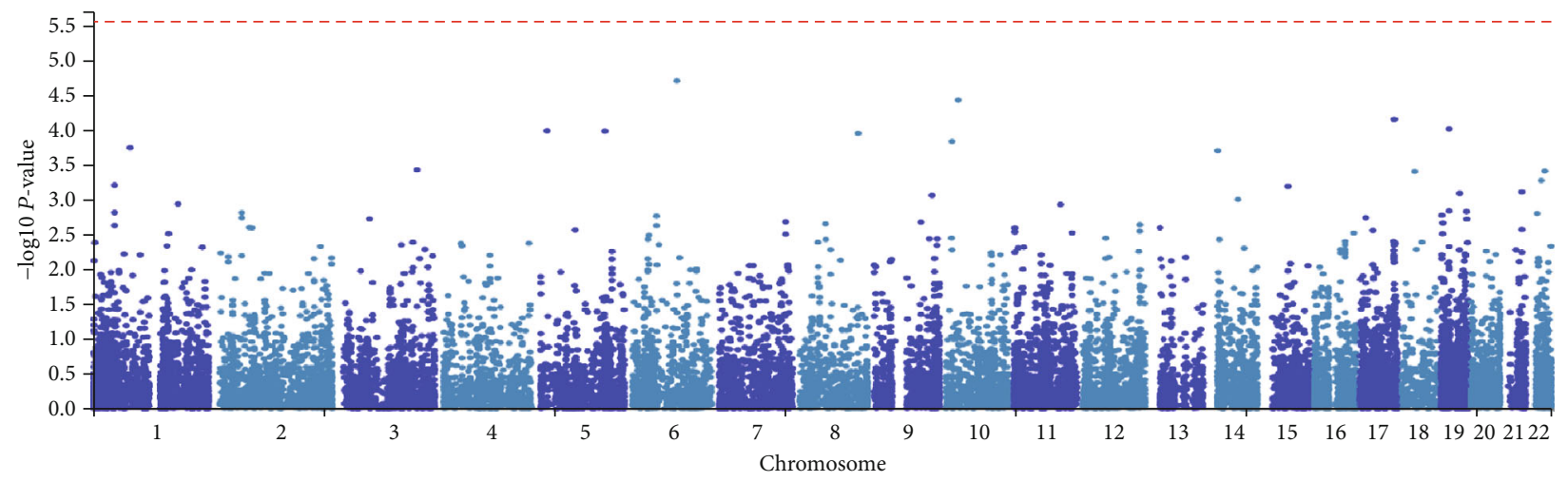

(a)

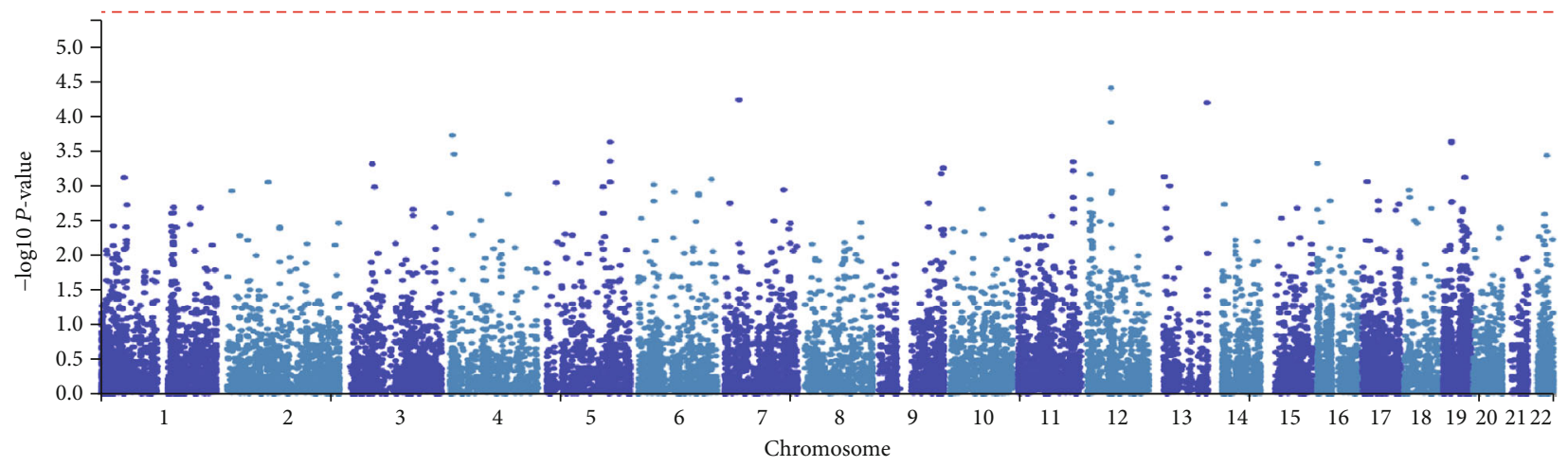

(b)

Figure 2: Manhattan plot of the gene-based test based on response to raloxifene. The red line represented suggestive significant threshold $\left(P=2.73 \times 10^{-6}\right)$ : (a) Lumbar spine site and (b) Femoral neck site.

TABLE 1: Top 10 significant genes of gene-based analysis at the lumbar spine site.

\begin{tabular}{lccccccc}
\hline Gene & CHR & START & STOP & Number of SNPs & ZSTAT & $p$ value & KO mouse bone phenotype $*$ \\
\hline FUT9 & 6 & 96463860 & 96663488 & 474 & 4.11 & $2.0 . E-05$ & NA \\
APBB1IP & 10 & 26727132 & 26856732 & 304 & 3.962 & $3.7 . E-05$ & NA \\
MYO15B & 17 & 73584139 & 73622929 & 104 & 3.808 & $7.0 . E-05$ & NA \\
USHBP1 & 19 & 17359985 & 17393595 & 54 & 3.728 & $9.7 . E-05$ & Vertebral fusion \\
DNAH5 & 5 & 13690440 & 13944652 & 691 & 3.712 & $1.0 . E-04$ & No \\
NRG2 & 5 & 139226364 & 139422884 & 238 & 3.709 & $1.0 . E-04$ & NA \\
KIAA0196 & 8 & 126036502 & 126104082 & 211 & 3.691 & $1.1 . E-04$ & NA \\
PHYH & 10 & 13319796 & 13344412 & 70 & 3.625 & $1.4 . E-04$ & No \\
ELTD1 & 1 & 79355449 & 79472403 & 309 & 3.567 & $1.8 . E-04$ & NA \\
OR4K1 & 14 & 20403767 & 20404842 & 7 & 3.545 & $2.0 . E-04$ & NA \\
\hline
\end{tabular}

CHR: chromosome; START: start position of the gene; STOP: stop position of the gene. $*$ Knockout mouse bone-related phenotypes were queried from International Mouse Phenotype Consortium.

and its binding protein are lower than those in the healthy individuals [26]. Thus, IGF-1 is critical in bone remodeling. The lower level of IGF-1 may increase the risk of hip fracture [27]. Taken together, iron overload may increase bone resorption and decrease bone formation through osteoblast and osteoclast activity or through the endocrine growth hormone(GH-) IGF-1 axis pathway.

Heilberg et al. reported that the rs9340799 (Xbal) AA genotype and rs2234693 (PvuII) CC genotype of the ESR1 have higher LS bone density after one-year treatment of raloxifene in Hispanic women [14]. Conversely, Mondockova et al. revealed that the AA genotype of rs9340799 and TT genotype of rs2234693 showed worse treatment response to raloxifene at the LS site in Slovak women [13]. Because of the inconsistent findings, we tried to confirm it by using this study. However, none of the variants were associated with the response to raloxifene in the current study. In another study, individuals with the CC genotype of the missense SNP 
TABLE 2: Top 10 significant genes of gene-based analysis at the femoral neck site.

\begin{tabular}{|c|c|c|c|c|c|c|c|}
\hline Gene & $\mathrm{CHR}$ & START & STOP & Number of SNPs & ZSTAT & $p$ value & KO mouse bone phenotype* \\
\hline AC068987.1 & 12 & 52203789 & 52206636 & 1 & 3.953 & $3.90 E-05$ & NA \\
\hline GHRHR & 7 & 30978284 & 31032869 & 139 & 3.859 & $5.70 E-05$ & BMD decrease \\
\hline F10 & 13 & 113777128 & 113803843 & 35 & 3.836 & $6.20 E-05$ & NA \\
\hline SCN8A & 12 & 51984050 & 52206648 & 200 & 3.674 & $1.20 E-04$ & NA \\
\hline TMEM128 & 4 & 4237269 & 4249950 & 84 & 3.562 & $1.80 E-04$ & NA \\
\hline MRPL34 & 19 & 17403418 & 17417652 & 35 & 3.51 & $2.20 E-04$ & NA \\
\hline HSPA9 & 5 & 137890571 & 137911133 & 14 & 3.501 & $2.30 E-04$ & NA \\
\hline ABHD8 & 19 & 17402940 & 17421045 & 42 & 3.494 & $2.40 E-04$ & BMD decrease \\
\hline ABLIM2 & 4 & 7967039 & 8160559 & 488 & 3.394 & $3.40 E-04$ & NA \\
\hline TMPRSS6 & 22 & 37461476 & 37505603 & 97 & 3.38 & $3.60 E-04$ & BMD decrease \\
\hline
\end{tabular}

CHR: chromosome; START: start position of the gene; STOP: stop position of the gene. $*$ Knockout mouse bone-related phenotypes were queried from International Mouse Phenotype Consortium.

TABLE 3: Gene-set analysis of raloxifene response at lumbar spine site.

\begin{tabular}{|c|c|c|c|c|c|c|}
\hline Database & Pathway & Number of genes & Beta & SE & $p$ value & $q$ value \\
\hline GO bp & Iron ion homeostasis & 63 & 0.363 & 0.101 & $2.00 E-04$ & 0.884 \\
\hline GO bp & Regulation of immunoglobulin secretion & 14 & 0.774 & 0.227 & $3.00 E-04$ & 0.884 \\
\hline GO bp & Transition metal ion homeostasis & 96 & 0.267 & 0.08 & $4.00 E-04$ & 0.884 \\
\hline Curated gene sets & $\begin{array}{l}\text { Inhibition of replication initiation } \\
\text { of damaged DNA by rb1 e2f1 }\end{array}$ & 10 & 0.879 & 0.266 & $5.00 E-04$ & 0.884 \\
\hline Curated gene sets & Apoptosis & 82 & 0.28 & 0.086 & $6.00 E-04$ & 0.884 \\
\hline GO bp & Response to protozoan & 20 & 0.569 & 0.177 & $6.00 E-04$ & 0.884 \\
\hline Curated gene sets & Cdh1 targets $3 \mathrm{dn}$ & 58 & 0.331 & 0.106 & $9.00 E-04$ & 0.884 \\
\hline Curated gene sets & Treating iron overload & 7 & 0.879 & 0.283 & $9.00 E-04$ & 0.884 \\
\hline GO bp & Toll like receptor signaling pathway & 77 & 0.3 & 0.099 & $1.00 E-03$ & 0.884 \\
\hline GO bp & Cellular iron ion homeostasis & 40 & 0.374 & 0.124 & $1.00 E-03$ & 0.884 \\
\hline Curated gene sets & Tlx targets $60 \mathrm{hr} \mathrm{dn}$ & 258 & 0.148 & 0.049 & $1.00 E-03$ & 0.884 \\
\hline GO bp & Negative regulation of platelet activation & 17 & 0.626 & 0.211 & $2.00 E-03$ & 0.884 \\
\hline Curated gene sets & Tgfb1 signaling via nfic $10 \mathrm{hr} \mathrm{dn}$ & 29 & 0.464 & 0.158 & $2.00 E-03$ & 0.884 \\
\hline Curated gene sets & Response to tosedostat $24 \mathrm{hr} \mathrm{dn}$ & 936 & 0.074 & 0.025 & $2.00 E-03$ & 0.884 \\
\hline Curated gene sets & Metabolism of amino acids and derivatives & 186 & 0.166 & 0.057 & $2.00 E-03$ & 0.884 \\
\hline GO bp & Cellular defense response & 54 & 0.323 & 0.111 & $2.00 E-03$ & 0.884 \\
\hline Curated gene sets & Akt phosphorylates targets in the cytosol & 12 & 0.559 & 0.193 & $2.00 E-03$ & 0.884 \\
\hline Curated gene sets & Asbcell pathway & 9 & 0.783 & 0.271 & $2.00 E-03$ & 0.884 \\
\hline Curated gene sets & Cell cycle s & 152 & 0.193 & 0.068 & $2.00 E-03$ & 0.884 \\
\hline Curated gene sets & Ar tf pathway & 47 & 0.352 & 0.124 & $2.00 E-03$ & 0.884 \\
\hline
\end{tabular}

GO: gene ontology; bp: biological process; $q$ values were from false discovery rate.

(Ala173Gly) of UGT1A8 revealed more improvement in negative symptoms after raloxifene treatment [17]. However, this variant cannot be replicated in the current study as well. We found that the most significant association with drug response at LS site was an intronic SNP of fucosyltransferase 9 (FUT9), rs7768089. This gene is a member of the glycosyltransferase family, and it participates in the biosynthesis of Lewis antigen. HaploReg v4.1 demonstrated that rs7768089 may alter the transcription factor (Foxa disc2, Nrd-2 3, and
TCF11::MafG) binding affinity, and according to GTEx, this SNP is an expression quantitative locus (eQTL) of FUT9 in the pancreas and brain tissue (S Table 3). However, the mechanistic relationship between FUT9 and BMD remains to be clarified.

For the FN site, the most significant SNP (rs34311394) was located within the intron between CSRP2 and E2F7. CSRP2 (cysteine and glycine rich protein 2) is involved in development and cellular differentiation, and E2F7 (E2F 
TABLE 4: Gene-set analysis of raloxifene response at femoral neck site.

\begin{tabular}{|c|c|c|c|c|c|c|}
\hline Database & Pathway & Number of genes & Beta & SE & $p$ value & $q$ value \\
\hline GO bp & Platelet morphogenesis & 16 & 0.762 & 0.208 & $1.00 E-04$ & 0.859 \\
\hline GO bp & $\begin{array}{l}\text { Regulation of nuclear transcribed mrna catabolic } \\
\text { process deadenylation-dependent decay }\end{array}$ & 14 & 0.633 & 0.187 & $3.00 E-04$ & 0.859 \\
\hline GO bp & Cellular modified amino acid catabolic process & 17 & 0.616 & 0.185 & $4.00 E-04$ & 0.859 \\
\hline Curated gene sets & Osteoblast differentiation by phenylamil up & 12 & 0.779 & 0.234 & $4.00 E-04$ & 0.859 \\
\hline GO bp & $\begin{array}{l}\text { Regulation of pri mirna transcription from rna } \\
\text { polymerase ii promoter }\end{array}$ & 16 & 0.691 & 0.209 & $5.00 E-04$ & 0.859 \\
\hline GO bp & $\begin{array}{l}\text { Regulation of establishment of protein } \\
\text { localization to mitochondrion }\end{array}$ & 118 & 0.234 & 0.073 & $7.00 E-04$ & 0.966 \\
\hline GO bp & Regulation of protein targeting to mitochondrion & 89 & 0.266 & 0.083 & $7.00 E-04$ & 0.966 \\
\hline Curated gene sets & Werner syndrome and normal aging up & 91 & 0.251 & 0.08 & $9.00 E-04$ & 0.991 \\
\hline Curated gene sets & Aml methylation cluster $6 \mathrm{dn}$ & 32 & 0.418 & 0.137 & $1.00 E-03$ & 0.999 \\
\hline GO bp & Epithelial structure maintenance & 21 & 0.559 & 0.184 & $1.00 E-03$ & 0.999 \\
\hline GO bp & $\begin{array}{l}\text { Regulation of substrate adhesion-dependent } \\
\text { cell spreading }\end{array}$ & 41 & 0.379 & 0.129 & $2.00 E-03$ & 0.999 \\
\hline Curated gene sets & Proteolytic cleavage of snare complex proteins & 16 & 0.752 & 0.26 & $2.00 E-03$ & 0.999 \\
\hline GO bp & Cardiac myofibril assembly & 16 & 0.574 & 0.204 & $2.00 E-03$ & 0.999 \\
\hline GO bp & $\begin{array}{l}\text { Positive regulation of pri mirna transcription } \\
\text { from rna polymerase ii promoter }\end{array}$ & 10 & 0.702 & 0.25 & $2.00 E-03$ & 0.999 \\
\hline Curated gene sets & Botulinum neurotoxicity & 18 & 0.674 & 0.241 & $3.00 E-03$ & 0.999 \\
\hline GO bp & Negative chemotaxis & 38 & 0.391 & 0.142 & $3.00 E-03$ & 0.999 \\
\hline GO bp & Regulation of endocrine process & 44 & 0.348 & 0.126 & $3.00 E-03$ & 0.999 \\
\hline Curated gene sets & Chondroitin sulfate biosynthesis & 17 & 0.507 & 0.184 & $3.00 E-03$ & 0.999 \\
\hline GO bp & Cellular metabolic compound salvage & 35 & 0.36 & 0.131 & $3.00 E-03$ & 0.999 \\
\hline GO bp & Cytokinesis & 80 & 0.246 & 0.09 & $3.00 E-03$ & 0.999 \\
\hline
\end{tabular}

GO: gene ontology; bp: biological process; $q$ values were from false discovery rate.

transcription factors) participates in the regulation of cell cycle progression. This SNP is associated with the expression of CSRP2 in the brain tissue (S Table 3). The most significant association revealed by a gene-based study was GHRHR (growth hormone-releasing hormone receptor). Although no traits related to $\mathrm{BMD}$ or drug response have been reported, the Ghrhr ${ }^{\text {tml.1(KOMP)Vlcg }}$ mouse model showed decreased bone mineral density according to the IMPC database. Based on the results in GWAS catalog, the genetic variants of GHRHR associated with congenital left sided heart lesions and adolescent idiopathic scoliosis. Further functional studies are needed to determine the plausible impact of these candidate genes on the bone.

Due to its estrogenic effects, raloxifene may increase the risk of thromboembolic events, such as deep vein thrombosis and pulmonary embolism. Estrogen increases the concentration of plasma fibrinogen and coagulation factors and enhances the aggregation of platelets [28]. From the gene-set analysis for the femoral neck site, the most significant treatment-associated gene-set was platelet morphogenesis. These results hint that genetic variants could possibly play a role in the occurrence of side effects. Thus, further studies may focus on the pharmacogenomics of thromboembolic events in raloxifene users. The main limitation of the current study is the sample size which is not able to confer sufficient statistical power to reach genome-wide significance. Larger cohorts are needed to replicate our results. Another limitation is the in the study. The majority of subjects are elderly patients with a mean age of 72.4 years. Previous studies have reported that bone loss may accelerate in the individuals who are over 70 years old [29]. In addition, aging influences GH and IGF1 serum levels [27], which might result in the change of BMD levels and the pharmacological effects. Further studies should also consider the age, the concentration of raloxifene in the blood, and the drug compliance for each patient.

\section{Conclusions}

In summary, we found plausible gene sets in iron ion homeostasis, osteoblast differentiation, and platelet morphogenesis pathways that may influence raloxifene responses. These findings offer new important insights and warrant further investigations into the pharmacogenomics of raloxifene.

\section{Data Availability}

The data used to support the findings of this study are included within the article. 


\section{Ethical Approval}

The study was approved by the Joint Institutional Review Board of Taipei Medical University (201210039).

\section{Consent}

All subjects provided written informed consent. All subjects provided written informed consent.

\section{Conflicts of Interest}

The authors declare that they have no conflict of interest.

\section{Authors' Contributions}

Wei-Chiao Chang and Kuo-Sheng Hung are responsible for the experimental design. Hsing-Fang Lu and Wan-Hsuan Chou performed the implementation of experiments. Hsing-Fang $\mathrm{Lu}$ analyzed the data. Kuo-Sheng Hung, PoHsin Chou, Shih-Tien Wang, and Wei-Chiao Chang are involved in the contribution of reagents/materials/analysis tools. Wei-Chiao Chang, Hsing-Fang Lu, Gan-Hong Lin, Wirawan Adikusuma, and Eko Mugiyanto interpreted the data. Kuo-Sheng Hung, Po-Hsin Chou, Shih-Tien Wang, and Hsing-Fang Lu are responsible for the clinical samples and data collection. Hsing-Fang $\mathrm{Lu}$ and Wei-Chiao Chang wrote the paper.

\section{Acknowledgments}

This work was supported by grants from the Ministry of Science and Technology in Taiwan (MOST 105-2628-B-038001-MY4 and MOST 106-2923-B-038-002) and the Higher Education Sprout Project by the Ministry of Education, Taiwan (MOE DP2-107-21121-A-05).

\section{Supplementary Materials}

Figure S1: evaluation of population stratification by principal component analysis. Figure S2: genome-wide association of response to raloxifene at lumbar spine bone mineral density. Figure S3: genome-wide association of response to raloxifene at femoral neck bone mineral density. Figure S4: $P$ value of ESR region $\pm 500 \mathrm{Mb}$ from response to raloxifene at LS site GWAS. Figure S5: $P$ value of UGT1A8 region $\pm 500 \mathrm{Mb}$ from response to raloxifene at LS site GWAS. Figure S6: $P$ value of ESR region $\pm 500 \mathrm{Mb}$ from response to raloxifene at $\mathrm{FN}$ site GWAS. Figure S7: $P$ value of UGT1A8 region $\pm 500 \mathrm{Mb}$ from response to raloxifene at FN site GWAS. Table S1: baseline characteristics of inclusion individuals. Table S2: variants reported to be related to raloxifene response previously Table S3: eQTL evidence of most significant SNPs. (Supplementary Materials)

\section{References}

[1] M. Lorentzon and S. R. Cummings, "Osteoporosis: the evolution of a diagnosis," Journal of Internal Medicine, vol. 277, no. 6, pp. 650-661, 2015.
[2] P. H. Chen, M. S. Lin, T. J. Huang, and M. Y. Chen, "Prevalence of and factors associated with adopting bone health promoting behaviours among people with osteoporosis in Taiwan: a cross-sectional study," BMJ Open, vol. 7, no. 9, article e015980, 2017.

[3] G. Hendrickx, E. Boudin, and W. Van Hul, "A look behind the scenes: the risk and pathogenesis of primary osteoporosis," Nature Reviews Rheumatology, vol. 11, no. 8, pp. 462-474, 2015.

[4] K. Estrada, U. Styrkarsdottir, E. Evangelou et al., "Genomewide meta-analysis identifies 56 bone mineral density loci and reveals 14 loci associated with risk of fracture," Nature Genetics, vol. 44, no. 5, pp. 491-501, 2012.

[5] H.-. F. Lu, K.-S. Hung, H.-W. Chu et al., "Meta-analysis of genome-wide association studies identifies three loci associated with stiffness index of the calcaneus," Journal of Bone and Mineral Research, vol. 34, no. 7, pp. 1275-1283, 2019.

[6] I. Kou, A. Takahashi, T. Urano et al., "Common variants in a novel gene, FONG on chromosome 2q33.1 confer risk of osteoporosis in Japanese," PLoS One, vol. 6, no. 5, article e19641, 2011.

[7] H.-F. Lu, K.-S. Hung, Y.-W. Hsu et al., “Association study between the FTCDNL1 (FONG) and susceptibility to osteoporosis," PLoS One, vol. 10, no. 10, article e0140549, 2015.

[8] N. C. Wright, A. C. Looker, K. G. Saag et al., "The recent prevalence of osteoporosis and low bone mass in the United States based on bone mineral density at the femoral neck or lumbar spine," Journal of Bone and Mineral Research, vol. 29, no. 11, pp. 2520-2526, 2014.

[9] D. Weycker, X. Li, R. Barron, R. Bornheimer, and D. Chandler, "Hospitalizations for osteoporosis-related fractures: economic costs and clinical outcomes," Bone Reports, vol. 5, pp. 186-191, 2016.

[10] S. H. Tella and J. C. Gallagher, "Prevention and treatment of postmenopausal osteoporosis," The Journal of Steroid Biochemistry and Molecular Biology, vol. 142, pp. 155-170, 2014.

[11] S. R. Cummings, S. Eckert, K. A. Krueger et al., "The effect of raloxifene on risk of breast cancer in postmenopausal women," JAMA, vol. 281, no. 23, pp. 2189-2197, 1999.

[12] P. D. Delmas, N. H. Bjarnason, B. H. Mitlak et al., "Effects of raloxifene on bone mineral density, serum cholesterol concentrations, and uterine endometrium in postmenopausal women," New England Journal of Medicine, vol. 337, no. 23, pp. 1641-1647, 1997.

[13] V. Mondockova, M. Adamkovicova, M. Lukacova et al., "The estrogen receptor 1 gene affects bone mineral density and osteoporosis treatment efficiency in Slovak postmenopausal women," BMC Medical Genetics, vol. 19, no. 1, p. 174, 2018.

[14] I. P. Heilberg, E. Hernandez, E. Alonzo et al., "Estrogen receptor (ER) gene polymorphism may predict the bone mineral density response to raloxifene in postmenopausal women on chronic hemodialysis," Renal Failure, vol. 27, no. 2, pp. 155$161,2009$.

[15] D. C. Kemp, P. W. Fan, and J. C. Stevens, "Characterization of raloxifene glucuronidation in vitro: contribution of intestinal metabolism to presystemic clearance," Drug Metabolism and Disposition, vol. 30, no. 6, pp. 694-700, 2002.

[16] D. Sun, N. R. Jones, A. Manni, and P. Lazarus, "Characterization of raloxifene glucuronidation: potential role of UGT1A8 genotype on raloxifene metabolism in vivo," Cancer Prevention Research, vol. 6, no. 7, pp. 719-730, 2013. 
[17] J. Labad, L. Martorell, E. Huerta-Ramos et al., "Pharmacogenetic study of the effects of raloxifene on negative symptoms of postmenopausal women with schizophrenia: a doubleblind, randomized, placebo-controlled trial," European Neuropsychopharmacology, vol. 26, no. 10, pp. 1683-1689, 2016.

[18] J. M. Kwon and A. M. Goate, "The candidate gene approach," Alcohol Research \& Health, vol. 24, no. 3, pp. 164-168, 2000.

[19] L. E. Duncan, M. Ostacher, and J. Ballon, "How genome-wide association studies (GWAS) made traditional candidate gene studies obsolete," Neuropsychopharmacology, vol. 44, no. 9, pp. 1518-1523, 2019.

[20] S. Purcell, B. Neale, K. Todd-Brown et al., "PLINK: a tool set for whole-genome association and population-based linkage analyses," American Journal of Human Genetics, vol. 81, no. 3, pp. 559-575, 2007.

[21] R. J. Pruim, R. P. Welch, S. Sanna et al., "LocusZoom: regional visualization of genome-wide association scan results," Bioinformatics, vol. 26, no. 18, pp. 2336-2337, 2010.

[22] K. Wang, M. Li, and H. Hakonarson, "ANNOVAR: functional annotation of genetic variants from high-throughput sequencing data," Nucleic Acids Research, vol. 38, no. 16, pp. e164e164, 2010.

[23] C. A. de Leeuw, J. M. Mooij, T. Heskes, and D. Posthuma, "MAGMA: generalized gene-set analysis of GWAS data," PLoS Computational Biology, vol. 11, no. 4, article e1004219, 2015.

[24] E. Balogh, G. Paragh, and V. Jeney, "Influence of iron on bone homeostasis," Pharmaceuticals, vol. 11, no. 4, p. 107, 2018.

[25] V. Jeney, "Clinical impact and cellular mechanisms of Iron overload-associated bone loss," Frontiers in Pharmacology, vol. 8, no. 77, 2017.

[26] A. Gaudio, N. Morabito, A. Catalano, R. Rapisarda, A. Xourafa, and A. Lasco, "Pathogenesis of thalassemia major-associated osteoporosis: a review with insights from clinical experience," Journal of Clinical Research in Pediatric Endocrinology, vol. 11, no. 2, pp. 110-117, 2019.

[27] P. P. M. M. Geusens and S. Boonen, "Osteoporosis and the growth hormone-insulin-like growth factor axis," Hormone Research, vol. 58, no. 3, pp. 49-55, 2002.

[28] J. Bonnar, "Coagulation effects of oral contraception," American Journal of Obstetrics and Gynecology, vol. 157, no. 4, pp. 1042-1048, 1987.

[29] C. Berger, L. Langsetmo, L. Joseph et al., "Change in bone mineral density as a function of age in women and men and association with the use of antiresorptive agents," CMAJ, vol. 178, no. 13, pp. 1660-1668, 2008. 